\title{
Erratum to: QTL mapping of carrot resistance to leaf blight with connected populations: stability across years and consequences for breeding
}

\author{
V. Le Clerc ${ }^{1} \cdot$ S. Marques ${ }^{1} \cdot$ A. Suel ${ }^{1} \cdot$ S. Huet ${ }^{1} \cdot$ L. Hamama $^{1} \cdot$ L. Voisine $^{1}$. \\ E. Auperpin ${ }^{1} \cdot$ M. Jourdan ${ }^{1}$ L. Barrot ${ }^{2}$ R. Prieur ${ }^{3}$ M. Briard ${ }^{1}$
}

Published online: 18 May 2016

C) Springer-Verlag Berlin Heidelberg 2016

\section{Erratum to: Theor Appl Genet (2015) 128:2177-2187 \\ DOI 10.1007/s00122-015-2576-z}

Unfortunately, the last column data of Table 2 of the above mentioned article was incorrectly published in the original version. The complete corrected Table 2 is given in the following page.

The online version of the original article can be found under doi:10.1007/s00122-015-2576-z.

V. Le Clerc

valerie.leclerc@agrocampus-ouest.fr

1 Agrocampus-Ouest, UMR 1345 Institut de Recherche en Horticulture et Semences, SFR 4207 QUASAV, 42 rue Georges Morel, 49071 Beaucouze Cedex, France

2 VILMORIN, Centre de recherche La Costière, 30210 Ledenon, France

3 HMCLAUSE, 1 Chemin du Moulin des Ronzières, 49800 La Bohalle, France 
Table 2 QTLs detected in single or connected populations in Les Landes

\begin{tabular}{|c|c|c|c|c|c|c|c|c|c|c|}
\hline \multirow[t]{2}{*}{$\begin{array}{l}\text { Scoring } \\
\text { year }\end{array}$} & \multirow[t]{2}{*}{ Population } & \multirow[t]{2}{*}{$\begin{array}{l}\text { Code of the } \\
\text { QTL }^{\mathrm{a}}\end{array}$} & \multirow[t]{2}{*}{$\begin{array}{l}\text { 1.5 LOD support } \\
\text { interval }(\mathrm{cM})^{\mathrm{b}}\end{array}$} & \multirow[t]{2}{*}{$\begin{array}{l}\text { Position } \\
\text { (cM) }\end{array}$} & \multirow[t]{2}{*}{$R^{2}(\%)$} & \multirow[t]{2}{*}{$\begin{array}{l}\text { Global } \\
R^{2}(\%)\end{array}$} & \multirow[t]{2}{*}{$F$ test } & \multicolumn{3}{|c|}{$\begin{array}{l}\text { Additive effect } \\
\text { of allele }\end{array}$} \\
\hline & & & & & & & & H1 & K3 & I2 \\
\hline \multirow[t]{13}{*}{2011} & \multirow[t]{3}{*}{ PC2 } & 1 & $0-22.4$ & 11.5 & 16.8 & \multirow[t]{3}{*}{37.7} & 6.2 & 0.23 & & -0.23 \\
\hline & & $4-1$ & $11.3-31.4$ & 23.9 & 16.9 & & 6.2 & -0.23 & & 0.23 \\
\hline & & $8-1$ & $8-59.6$ & 27.3 & 15.9 & & 5.8 & 0.20 & & -0.20 \\
\hline & \multirow[t]{3}{*}{ PC3 } & $6-1$ & $50.7-67.6$ & 56.2 & 28.9 & \multirow[t]{3}{*}{52.8} & 8.2 & 0.27 & -0.27 & \\
\hline & & $8-1$ & $35.5-39.1$ & 37.7 & 43.8 & & 13.3 & 0.34 & -0.34 & \\
\hline & & $9-1$ & $18.9-66.5$ & 29.8 & 13.6 & & 3.9 & 0.18 & -0.18 & \\
\hline & \multirow{7}{*}{$\begin{array}{l}\mathrm{PC} 2 \text { and PC3 con- } \\
\text { nected }\end{array}$} & 1 & $0-20.5$ & 10 & 19.2 & \multirow[t]{7}{*}{52.5} & 10.4 & 0.29 & 0.07 & -0.36 \\
\hline & & 2 & $34.1-49$ & 49 & 6.6 & & 3.3 & 0.11 & 0.06 & -0.17 \\
\hline & & $4-1$ & $10.5-27.4$ & 14.8 & 12.6 & & 6.6 & -0.21 & -0.00 & 0.21 \\
\hline & & 5 & $1.6-114.8$ & 28.9 & 4.8 & & 2.4 & 0.12 & 0.34 & -0.46 \\
\hline & & $6-1$ & $50.2-66.1$ & 57 & 16.0 & & 8.5 & 0.07 & -0.38 & 0.31 \\
\hline & & $8-1$ & $31.8-38.1$ & 35.1 & 27 & & 15.4 & 0.32 & -0.30 & -0.02 \\
\hline & & $9-1$ & $29.4-51.7$ & 40.2 & 8.7 & & 4.5 & 0.19 & -0.23 & 0.04 \\
\hline \multirow[t]{15}{*}{2014} & \multirow[t]{4}{*}{ PC2 } & 1 & $10.5-24.1$ & 17.2 & 34.8 & \multirow[t]{4}{*}{52.7} & 12.4 & 0.45 & & -0.45 \\
\hline & & $4-1$ & $10.5-39.6$ & 18.3 & 18 & & 6.1 & -0.28 & & 0.28 \\
\hline & & $8-2 ?$ & $2.7-58.8$ & 11 & 11 & & 3.8 & 0.20 & & -0.20 \\
\hline & & $9-2$ & $36.5-68.4$ & 52 & 12.1 & & 4.2 & 0.21 & & -0.21 \\
\hline & \multirow[t]{5}{*}{ PC3 } & $4-2$ & $29.4-41.3$ & 36.1 & 16.6 & \multirow[t]{5}{*}{51.7} & 4.4 & -0.20 & 0.20 & \\
\hline & & $6-1$ & $44.2-67.6$ & 60.1 & 14 & & 3.7 & 0.18 & -0.18 & \\
\hline & & $6-2$ & $0-15.9$ & 0.7 & 14 & & 3.8 & 0.15 & -0.15 & \\
\hline & & $8-1 ?$ & $26.1-62.9$ & 47 & 24 & & 6.4 & 0.24 & -0.24 & \\
\hline & & $9-2$ & $49.7-65$ & 59.4 & 18 & & 4.8 & 0.20 & -0.20 & \\
\hline & \multirow{6}{*}{$\begin{array}{l}\text { PC2 and PC3 con- } \\
\text { nected }\end{array}$} & 1 & $9.3-24.6$ & 20 & 23.1 & \multirow[t]{6}{*}{43} & 12.5 & 0.38 & 0.28 & -0.66 \\
\hline & & $4-1$ & $14.4-51$ & 19.5 & 14 & & 7.2 & -0.30 & -0.01 & 0.31 \\
\hline & & $4-2^{c}$ & $15.2-39.6$ & 34.5 & 10.6 & & 5.5 & -0.31 & 0.15 & 0.16 \\
\hline & & $8-1$ & $23.7-54.9$ & 30 & 10.8 & & 5.4 & 0.25 & -0.20 & -0.05 \\
\hline & & $8-2 ?$ & $2-52.5$ & 5.7 & 10.2 & & 5.2 & 0.28 & 0.01 & -0.29 \\
\hline & & $9-2$ & $51.3-69.7$ & 61.7 & 11.5 & & 5.8 & 0.27 & -0.17 & -0.1 \\
\hline
\end{tabular}

$R^{2}$ and global $R^{2}$ are the phenotypic part of the variation explained, respectively by one or all the QTLs detected at one scoring date

${ }^{a}$ Correspond to the number of the linkage group followed by a number when more than two QTLs were identified on the same linkage group

? Is added when affiliation is uncertain

${ }^{\mathrm{b}}$ LOD confidence intervals and positions correspond to the ones on consensus map

${ }^{c}$ QTLs in italic were not taken into account in the calcul of global $R^{2}$. QTLs identified with choice of manual cofactors 